\title{
Natural Frequency Testing and Model Correlation of Rocket Engine Structures in Liquid Hydrogen - Phase I, Cantilever Beam
}

\author{
Andrew M. Brown, Ph.D. ${ }^{\text {a }}$, Jennifer L. DeLessio ${ }^{\mathrm{b}}$, Preston W. Jacobs ${ }^{\mathrm{c}}$ \\ ${ }^{a}$ Aerospace Engineer, NASA/Marshall Space Flight Center, \\ ER41/Propulsion Structural \& Dynamic Analysis, Huntsville, AL 35812 \\ bPropulsion Systems Analyst, Jacobs-ESSSA - NASA/Marshall Space Flight Center, \\ ER41/Propulsion Structural \& Dynamic Analysis, Huntsville, AL 35812 \\ ${ }^{c}$ Materials Test Engineer, Jacobs-ESSSA - NASA/Marshall Space Flight Center, \\ EM22/ Materials Test, Chemistry, and Contamination Control Branch, Huntsville, AL 35812
}

\begin{abstract}
Many structures in the launch vehicle industry operate in liquid hydrogen (LH2), from the hydrogen fuel tanks through the ducts and valves and into the pump sides of the turbopumps. Calculating the structural dynamic response of these structures is critical for successful qualification of this hardware, but accurate knowledge of the natural frequencies is based entirely on numerical or analytical predictions of frequency reduction due to the addedfluid-mass effect because testing in LH2 has always been considered too difficult and dangerous. This fluid effect is predicted to be approximately $4-5 \%$ using analytical formulations for simple cantilever beams. As part of a comprehensive test/analysis program to more accurately assess pump inducers operating in LH2, a series of frequency tests in LH2 were performed at NASA/Marshall Space Flight Center's unique cryogenic test facility. These frequency tests are coupled with modal tests in air and water to provide critical information not only on the mass effect of LH2, but also the cryogenic temperature effect on Young's Modulus for which the data is not extensive. The authors are unaware of any other reported natural frequency testing in this media. In addition to the inducer, a simple cantilever beam was also tested in the tank to provide a more easily modeled geometry as well as one that has an analytical solution for the mass effect. This data will prove critical for accurate structural dynamic analysis of these structures, which operate in a highly-dynamic environment.
\end{abstract}

Keywords: Structural Dynamics, Modal Test, Liquid Hydrogen, Model Correlation, Modulus temperature dependence

\section{NOMENCLATURE}

$\begin{array}{ll}A_{m 1} & \text { added mass for beam-type modes } \\ a & \text { cantilever beam length } \\ b & \text { beam width } \\ \{\mathrm{f}\} & \text { Vector of Natural Frequencies } \\ {[\Phi]} & \text { Modal Matrix } \\ \text { DOF } & \text { Degrees of Freedom } \\ \text { DOE } & \text { Design of Experiments } \\ \text { LH2 } & \text { Liquid Hydrogen } \\ \text { LOX } & \text { Liquid Oxygen } \\ \text { LPFP } & \text { Low Pressure Fuel Pump } \\ \text { RT } & \text { Room Temperature } \\ \rho_{f} & \text { fluid mass density }\end{array}$




$\begin{array}{ll}\text { SAVPE } & \text { Sum of Absolute Values of Percent Errors } \\ \text { SSME } & \text { Space Shuttle Main Engine } \\ \omega_{\mathrm{f}} & \text { natural frequency of the beam immersed in fluid } \\ \omega_{\mathrm{v}} & \text { natural frequency of the beam in vacuum }\end{array}$

\section{INTRODUCTION}

Liquid rocket engines are powered by the combustion of two propellants at very high pressure, a fuel and an oxidizer. Frequently, the fuel is liquid hydrogen (LH2). While the pressure can be provided by a very high-strength storage tank, usually this would be weight-prohibitive, so a turbopump or series of turbopumps are required to provide these extremely high pressures. These rotating shafts of these turbopumps are usually driven by a hot gas in the turbine side, and the pumping side consists of either axial inducers, which increase the velocity by conically swept blades on the shaft, or centrifugal impellers, which force the fluid into spinning outward radiating channels; both these actions significantly increase the flow velocity, and high pressures are then obtain by diffusing the flow.

Because of a number of highly-energetic fluid excitation sources at both narrow-band frequency range or harmonic frequencies, a structural dynamic analysis is required for all the components in the flow path on both the turbine and pump sides in order to prevent high dynamic response. However, because of several complicating factors, this analysis is problematic. First, the components operate in a liquid, which not only provides the excitation forcing function but also affects both the damping and structural dynamic characteristics due to virtual mass loading (natural frequencies $\{\mathrm{f}\}$ and modes $[\Phi])$. Second, for liquid hydrogen pumps, the operating temperature is $-253^{\circ} \mathrm{C}\left(-423^{\circ} \mathrm{F}\right)$, which has a substantial effect on the Young's Modulus E of the metallic materials used for the components, and therefore affects the natural frequencies substantially.

This issue has always been a problem for the design of liquid hydrogen turbopumps, but it became much more of a risk during the design phase of the J-2X engine, from 2006-2012. The frequency range of one of the excitation sources, higher order cavitation, was predicted to overlap with the oxidizer pump inducer natural frequencies. This motivated an extensive analytical examination, which was forced to incorporate the large variability in the structural dynamic characteristics due to the large uncertainties discussed above, and a test program to characterize the environment with high-frequency pressure taps and structural response via strain gages in a water (not liquid oxygen) rig (see Figure 1). A great deal of important data was obtained including some information on damping and the mass loading effect of water on the inducer natural frequencies. The most challenging goal of fully anchoring the forcing function was not completed before the J-2X program was cancelled.

This same potential HOC issue existed during Space Shuttle Main Engine (SSME) and has arisen again during the implementation of the RS-25, which is an updated SSME engine for the core stage of NASA's new Space Launch System. The SSME had operated with some speed placards to avoid this resonant condition, but new operating requirements for the RS-25 meant the condition could not be avoided easily. An extensive analysis was performed, but due to the uncertainties discussed above, along with the uncertainties in the forcing function itself, the analysis predicted failure even for SSME operation, which testing and run-time in the millions of seconds had shown was not occurring. An empirically-based, non-physics-based damage-fraction analysis was therefore performed based upon one of the tested units, and an acceptance criteria stemming from this analysis were imposed upon the inducer and the operation of the turbopump. Some of the resulting restrictions are quite undesirable, though, and possibly could be severe if conditions show the levels of HOC are greater than expected.

A new integrated test/analysis program has been initiated, therefore, both to mitigate some of the restrictions of the criteria and to provide data for a physics-based analysis that would produce believable results. This integrated program includes an updated waterflow test, hydroelastic analysis and testing, acoustic modeling, and unique natural frequency testing of the sub-scale stainless-steel inducer used in the water-flow test in LH2. Along with more complete modal testing in air and water, this LH2 test will provide data necessary to correlate the inducer natural frequencies and validate analytical predictions of the structural characteristics in this environment. The LH2 testing 


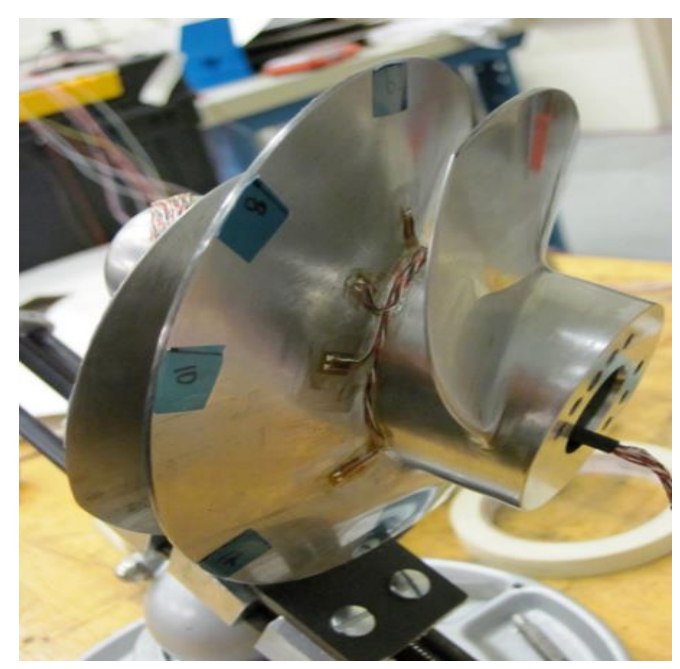

Fig. $1 \mathrm{~J}-2 \mathrm{X}$ LOX Turbopump Inducer (not to scale)

is enabled by the unique capabilities of the NASA/Marshall Space Flight Center Cryogenic Test Facility. The authors have not found documentation of modal testing in LH2 of any kind in the literature.

In addition to testing the inducer in LH2, an opportunity existed to perform a test of a much simpler "cantilever beam" structure. This test would allow very precise modeling of the exact physical structure, and allow comparison with purely analytical as well as numerical modal analyses. This test would also provide an opportunity to implement any lessons learned prior to testing the inducer. Here, we provide preliminary results and data on this LH2 "ping" test of the cantilever beam. Final documentation will be provided upon completion of both phases of the LH2 frequency test/analysis program and the entire inducer water-flow integrated test/analysis program.

\section{LITERATURE SURVEY}

The first aspect examined by this test/analysis program is the effect on the Young's Modulus of metals at cryogenic temperatures, in particular the Titanium alloy used for the RS-25 LPFP inducer. We examined a number of publically available sources, shown in Figure 2, including the well-known MMPDS [1], a recent hybrid test/analysis study by Ghisi and Mariani [2], and a dynamic modulus test by Zhang, et. al.[3] using a modal test of a cantilever beam in a cryostat, similar to the one performed in this study. We also have access to several proprietary sources, which cannot be reported here. The variability in this data resulted in a non-trivial uncertainty in the operating natural frequency of the inducer blades. We will discuss how this data was used in the results section of this document.

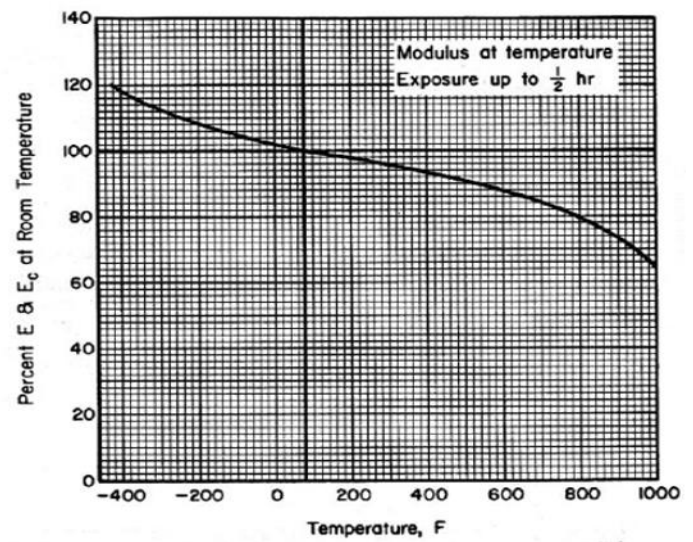

a.

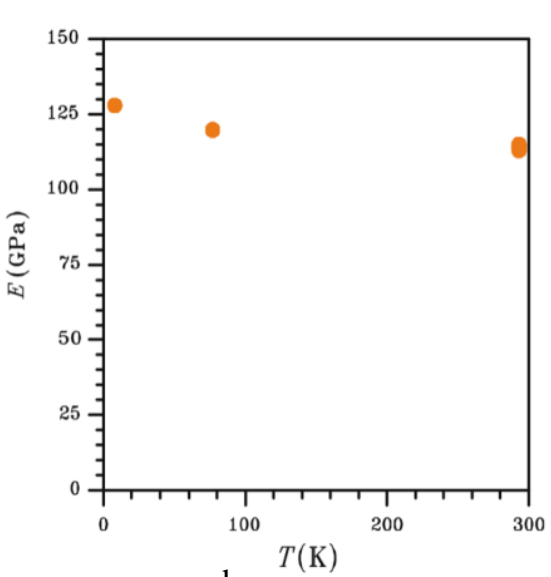

b.

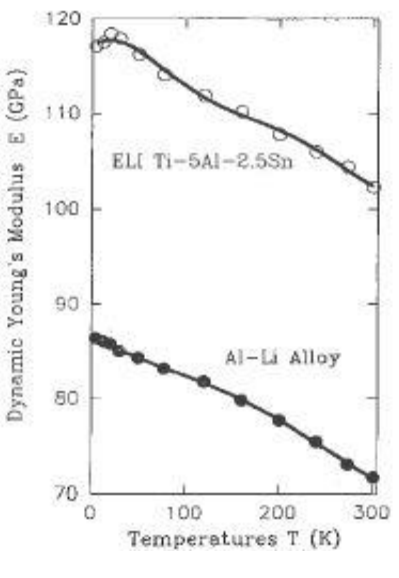

c.

Fig. 2 Public Data Relating Young's Modulus of Ti 5-2.5 to Temperature; a) MMPDS, b) Ghisi \& Mariani, c) Zhang 
The second aspect is the effect of the liquid mass of the LH2 on $\{\mathrm{f}\}$ and $[\Phi]$ of the inducers. There has been a great deal of both analytical and numerical work in this field beginning with Lindholm in 1965 [4] and continuing until the present. Although Lindholm's experiments were in water, as is the case for almost every other experimental study, he did generate an equation relating the density of the liquid to the natural frequencies, where the same equation is applicable for all cantilever beam-type modes, as derived below

$$
A_{m 1}=\frac{\pi}{4} \rho_{f} a b^{2}
$$

Where $A_{m l}$ is the added mass for beam-type modes, $\rho_{f}$ is fluid mass density, $a$ is the beam length, and $b$ is beam width. This added mass can then be used to calculate the ratio of the natural frequency of the beam immersed in fluid $\omega_{\mathrm{f}}$ to the natural frequency of the beam in vacuum $\omega_{\mathrm{v}}$ (or a low-density gas) using

$$
\frac{\omega_{f}}{\omega_{v}}=\frac{1}{\sqrt{1+\frac{A_{m 1}}{m_{b}}}} .
$$

Much of the published work deals with rectangular plates with low aspect ratios, (e.g., Ergin \& Ugurlu [5], Hosseini-Hashemi [6]) but some do examine longer beams which have aspect ratios on the order of 10 as used here, in particular Liang [7], who used previous experimental and analytical results to generate a correction factor for aspect ratio. Liang succinctly derives and identifies the important parameters as follows:

The added mass/unit span due to the fluid is

$$
A_{m 1}=0.25 C_{f} \frac{\pi \rho_{f} b}{\rho_{p} h_{p}}
$$

where $\rho_{f}$ is fluid mass density, $\rho_{p}$ is structural mass density, $b$ is beam width, $h_{p}$ is beam thickness, and

$$
\mathrm{C}_{f}=\frac{2 * \text { aspect_ratio }}{1+2 * \text { aspect_ratio }}
$$

These then can be directly used to calculate the natural frequency in the fluid using

$$
\omega_{f}=\omega_{v} \sqrt{\frac{1}{1+\frac{A_{m 1}}{C_{f}}}} .
$$

As far as other work published in the literature, many of these focus on numerical solutions to the problem (e.g. Kerboua [8]). As a high-fidelity multi-physics numerical code ANSYS ${ }^{\circledR}$ is already being used in this correlation effort, we do not believe it adds value to implement these solutions.

\section{LH2 PING TEST SETUP}

The test environment for the ping test is a large cryostat, essentially a cylindrical canister that can be filled with cryogenic fluids. The cryostat is bolted to a top section (Dewar Head) that contains an actuator that can move axially. Upon examination by the analysis/testing team, a simple and reliable test design and process was conceived. First, since LH2 is a very low-density fluid, a previous study by Chiu and Brown [9] indicated that the mode shapes would not vary perceptibly from the in-vacuo case, so only a natural frequency test, rather than a full modal test, was necessary since the $\{\mathrm{f}\}$ of interest could be tied to the correct $[\Phi]$ using previous in-vacuo modal analysis and modal testing in air. Second, since access to the filled cryostat would be difficult, the availability of an existing actuating mechanism within the cryostat could be taken advantage of by fabricating a "pick" (similar to a 
guitar pick) that could be lowered at a rate fast enough to pluck the structure and still get out the way fast enough to prevent a repeated impact. A photo of the cryostat in operation and the ping-test assembly is shown in Figure 3.

A titanium beam of aspect ratio 10 was then fabricated using a billet of material from the same batch as is used to construct the inducers. The closest producible reproduction of a theoretically fully-fixed end was to bolt the beam onto a stiff column, as shown in a subset of pre-test modal analysis results in Figure 3. Although pre-test analysis showed that the targeted modes were almost pure "cantilever" modes, as intended, the mounting column as well as the four bolts connecting it to the beam, including their pre-load, were modelled to capture the natural frequencies as accurately as possible. The influence of the mounting column is clear in the second mode, which is not one of the four targeted modes. In addition, four thermocouples were installed inside the cryostat to provide temperature measurements, and two LH2-compatible strain gages were installed on the upper and lower surfaces of the beam near the fixed end.

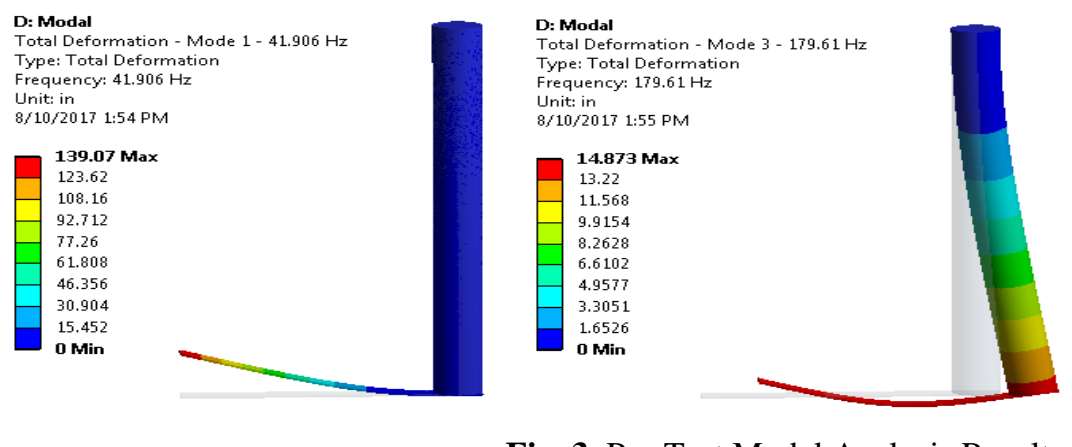

Fig. 3 Pre-Test Modal Analysis Results
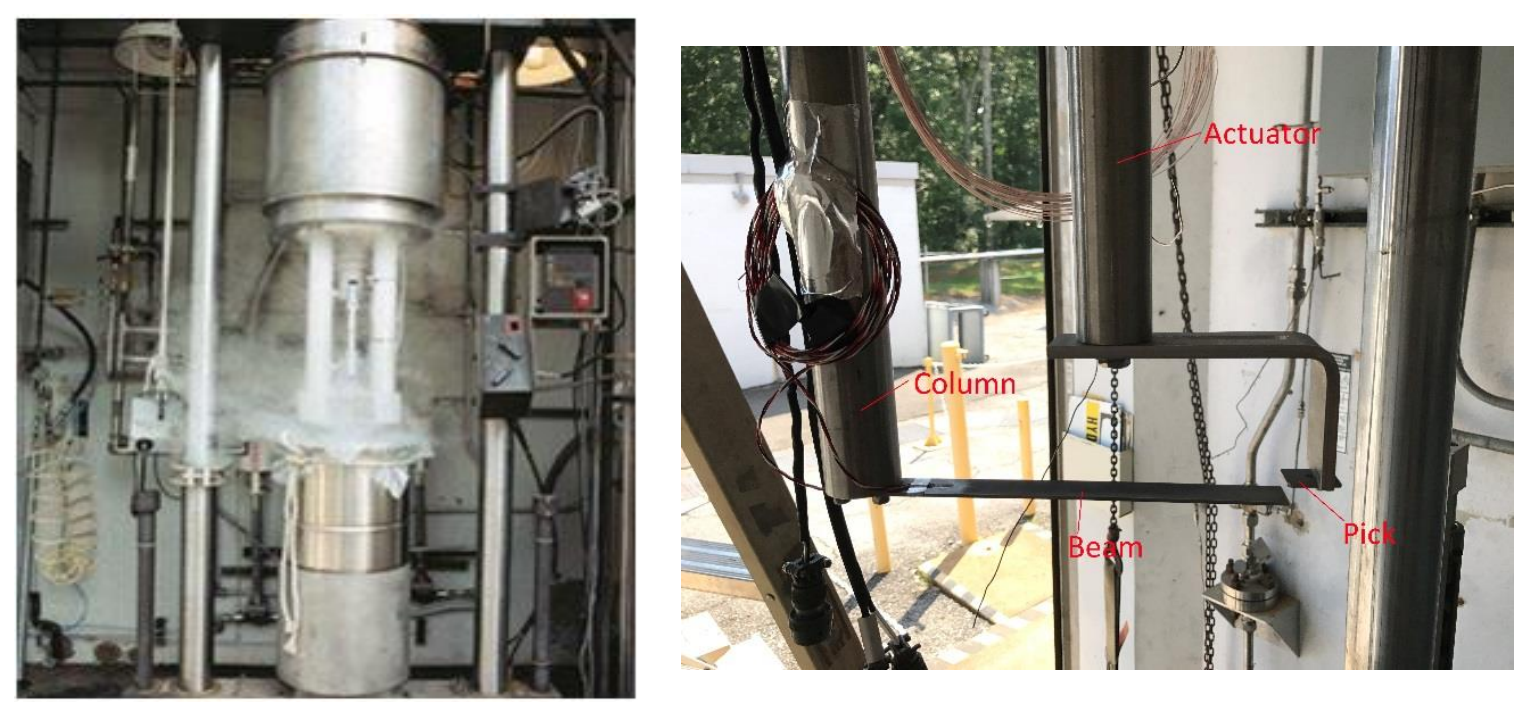

Fig. 4 Cryostat and Cantilever Beam Test Setup

\section{CORRELATION PROCEDURE}

Determining a well-defined correlation procedure a-priori is also critical for accurately assessing the validity of the models for predicting the effect of liquid mass and temperature on natural frequency. Of course, this procedure was adapted to the test results, and resulted in the following steps: 
1) Using optimization procedure, vary the room temperature (RT) Young's Modulus to best match the four measured natural frequencies, with the result being four new calculated frequencies. The error-function for the optimization is to minimize the sum of the absolute values of the percent errors (SAVPE) for all four tested beam bending modes.

2) If the resulting RT Modulus is within the range given by estimates from the available sources, use this Modulus and apply ANSYS numerical methods to determine predictions for frequencies in water and compare to measured frequencies. The purpose of this step was to calibrate the fluid modeling techniques in ANSYS. Although the results in water were considered estimates, the calculated results provided confidence in these tested values.

3) If the optimized RT Modulus is within the range given by estimates from the available sources, repeat for the Boil-off case.

4) Evaluate whether the optimized Modulus at Boil-off conditions is within the spread of available data. If so, extrapolate this Modulus to LH2 temperature and calculate the natural frequencies using the same method as step 2) and compare to measured frequencies. In addition, apply each of previously-discussed purely analytical techniques developed by Lindholm and Liang to obtain frequency predictions, and compare with ANSYS results.

\section{MEASUREMENT, MODELING AND NUMERICAL ANALYSIS OF MODAL TEST CONFIGURATION}

Engineering drawings used to fabricate the beam and column provided a starting point for generating the solid model geometry (in ANSYS SpaceClaim). Post-test, the volume and weight of the beam were precisely measured. The beam weight was measured on a calibrated scale, which read to four decimal places. The volume of the beam was measured using a graduated cylinder. In addition, precise measurements of the beam dimensions were taken with digital calipers. The length and width were reported and the thickness of the beam was reported every half inch. These measurements provided the data to construct a more accurate solid model in SpaceClaim and to also calculate the density of the material and compare to published values. The calculated density calculated was $4462.0 \mathrm{~kg} / \mathrm{m}^{3}\left(0.1612 \mathrm{lb} / \mathrm{in}^{3}\right)$, which compares well to available data. In order to match the as-measured mass of the beam, the density in the ANSYS finite element model (FEM) was defined such that:

$$
\begin{aligned}
& \rho_{\text {Meas }}=\mathrm{m}_{\text {Meas }} / \mathrm{V}_{\text {Meas }} \\
& \rho_{\text {Model }}=\left[\mathrm{V}_{\text {Meas }} / \mathrm{V}_{\text {model }}\right] * \rho_{\text {Meas }}=\mathrm{m}_{\text {Meas }} / \mathrm{V}_{\text {model }}
\end{aligned}
$$

ANSYS version 18.0 was used for all analysis. ANSYS SpaceClaim was used to construct the geometry, Mechanical was used for the pre-stressed modal solutions, and DesignXplorer was used for the optimization procedure. The Acoustics Application Customization Toolkit (ACT) extension was used in ANSYS Mechanical for the simulations with modeled fluids.

For the FEM models used in steps 2) and 4) above, the fluid volume was also constructed in SpaceClaim, as shown in Figure 5. An Enclosure was constructed around the beam and column using the dimensions of the cryostat and fluid level during test. Shared topology (merged nodes) were defined between the beam/fluid and the column/fluid interfaces, but the topology was not shared between the beam and the column. This is because nonlinear contact was used in the static pre-stress solution. Early modeling of the beam found that the inclusion of the column and the actual contact interface between the beam and the column were necessary to adequately model the stiffness of the structure. Initially the model included only the beam fixed at one end in all degrees of freedom (DOF). The column was then added and was initially fully bonded to the beam. The next and final configuration included the bolts at the beam/column interface with pretension, shown in Figure 6. As the model fidelity increased the modal results converged and the optimized RT Modulus increased and converged as well (final shapes shown in Figure 7). 


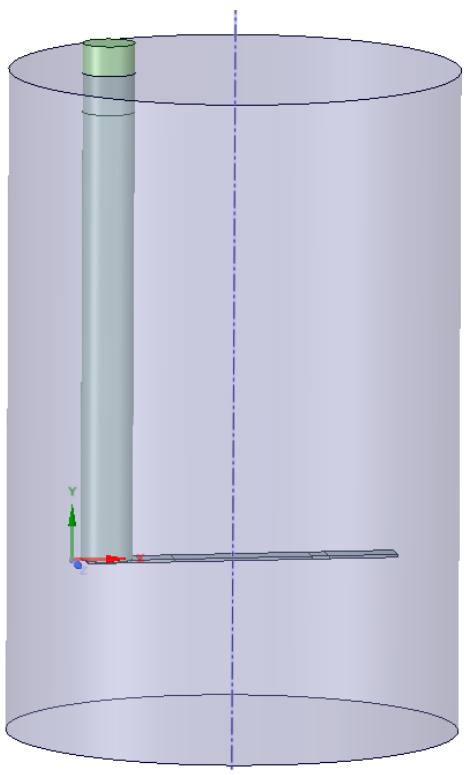

Fig. 5 SpaceClaim Model with Fluid

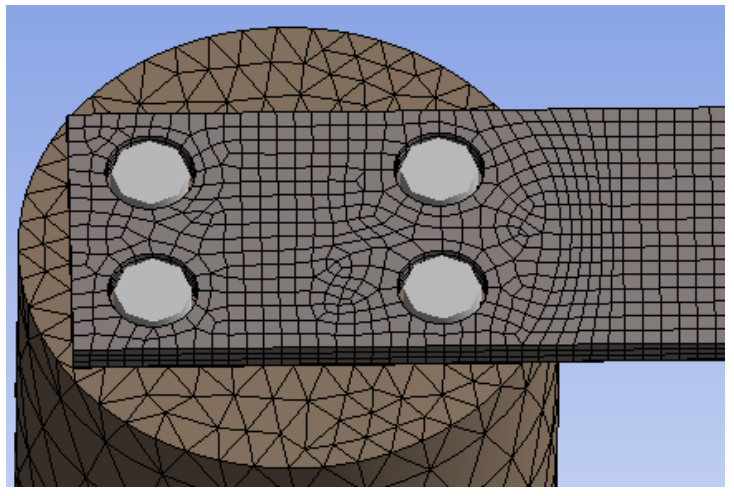

Fig. 6 View of ANSYS FEM Mesh

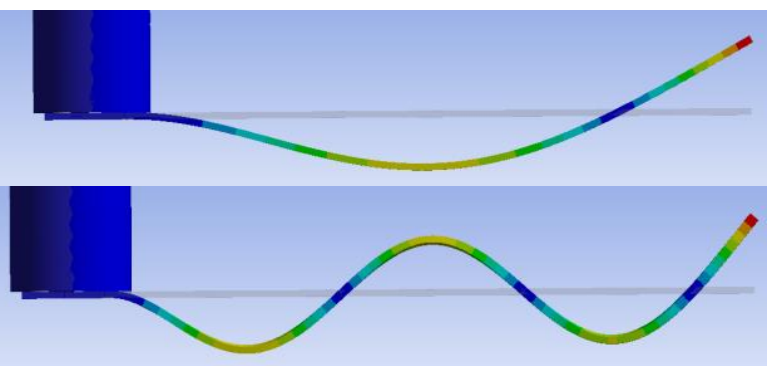

Fig. 7 Final Analytical Mode Shapes used for Correlation

The prestressed modal analysis was defined within ANSYS Mechanical. Beam Connections were created to define the bolts between the beam and the column. This connection type inserts beam elements which can have pretension applied. As the assembly torque was not measured, a reasonable preload force was defined for each bolt. The modal results were found to be relatively insensitive to the actual preload values (as long as a similar contact pattern was achieved.) The upper surface of the column (which attaches to the Dewar Head) was assumed fixed in all DOF. For the models with fluid elements, the acoustic body and fluid structure interfaces were defined in the static solution and the flag for "subsequent linear perturbation" analysis was defined. The water or LH2 density and speed of sound were defined for their respective temperatures.

The "True" contact status was used in the subsequent modal solution. The Unsymmetric solver was used for the modal analyses with fluid elements.

ANSYS DesignXplorer was used to determine optimal Modulus values in correlation steps 1) and 3). A Central Composite Design was completed with the Modulus as the single input parameter and the SAVPE as the single, custom-defined output parameter. A Genetic Aggregation response surface was developed and Screening Optimization was performed where SAVPE was minimized. 


\section{TESTING}

In order to isolate the effects of temperature and added liquid mass on natural frequency, as well as enabling recognition of existing sources of uncertainty, a well-defined testing procedure was necessary. The first step was a ping test of the beam setup under ambient conditions, where the air temperature was measured with thermocouples. There are four thermocouples in the cryostat, one at the surface of the fluid, one slightly above the beam, one slightly below, and one near the bottom of the cryostat. The beam temperature used for each test is the average of the thermocouples just above and just below. Three separate pings were performed for this (and every) step, and the time histories obtained and transformed into the frequency domain where the first four peaks could clearly be identified. Next, LH2 was slowly added to the cryostat, and when the entire apparatus was fully submerged with several inches of clearance from the top of the mounting cylinder, the next three ping tests were performed. The LH2 was then quickly removed by opening the liquid vent and filling with gaseous Helium, and three more pings were performed while the temperatures were still close to cryogenic ("Boil-off" configuration). Readings from the thermocouples were also taken. The data from this series of tests was also considered to be high quality. The next day, after the apparatus had completely warmed to ambient temperatures, a fourth series of ping tests was performed in water. Unfortunately, the strain gages never fully stabilized, perhaps due to a possible incompatibility with water, so the results from this water-test phase can only be considered "best estimates".

\section{RESULTS}

The complete results for the test and analysis series are shown in Table 1 to enable appropriate comparisons and error assessment. These should be considered preliminary, as they have not been through a quality control process yet, but the authors believe they will not be changed in any way. The first step in the modal correlation procedure involved optimizing the SAVPE error by adjusting the RT Modulus of the cantilever beam. The DOE bounds were reasonably defined based on the spread of available data at RT with some additional margin. The screening optimization produced three candidate points and the Modulus was selected based on the lowest SAVPE. This Modulus value aligned well with several of the propriety data sources. This same optimization approach was used for the Boil-off configuration. Each bending mode was matched to less than 0.5\% with an SAVPE value of 1.04\%. There was some concern in these results because the Modulus values obtained are significantly higher than those reported by Zhang, which had a very similar test setup. We believe that the discrepancies are most likely due to the fact that his team used analytical methodologies to predict the beam natural frequencies and assumed a fixed edge boundary condition. The fixed edge assumption over-predicts the frequencies, and analytical techniques frequently do so as well, so the calculated Young's Modulus would be too low.

The measured temperature at the beam location in the cryostat for the air RT and water tests was only two degrees different. Thus this same optimized RT Modulus value was applied in the model with the added water fluid elements as discussed in the modeling section. The results are excellent, with less than $1 \%$ error for each of the four modes compared with test and an SAVPE value of $2.58 \%$. In addition, the added mass frequency factor $\left(\omega_{\mathrm{f}} / \omega_{\mathrm{v}}\right)$ are calculated and shown for the test cases, the ANSYS models, and applying the analytical techniques from Lindholm and Liang, and these all show excellent agreement of less than $1 \%$ error.

Moving to the last step of the correlation procedure, since all available data indicates that the Modulus does not change between temperatures at the Boil-off configuration and LH2, the ANSYS fluid elements were added and the analytical techniques were applied to the model using the Boil-off optimized Modulus and compared with the LH2 test results. Again, the results are excellent, with frequency errors and errors in added mass frequency factors all less than $1 \%$ and an SAVPE error of $2.36 \%$. 
Table 1 Modal Test and Analysis Summary

\begin{tabular}{|c|c|c|c|c|c|c|c|c|c|c|c|c|c|c|}
\hline \multicolumn{4}{|c|}{ Air RT } & & & & & \multicolumn{5}{|c|}{ Boil-off and LH2 Temperature } & & \\
\hline $\begin{array}{l}\text { Mode } \\
\text { (Test } \\
\text { Order) }\end{array}$ & Test $(\mathrm{Hz})$ & \begin{tabular}{|l|} 
ANSYS \\
FEM \\
Opt- \\
imized E
\end{tabular} & $\%$ Error & & & & & Test $(\mathrm{Hz})$ & $\begin{array}{l}\text { ANSYS } \\
\text { FEM } \\
\text { Optimized } \\
\text { E (Hz) }\end{array}$ & \multicolumn{3}{|l|}{$\%$ Error } & & \\
\hline 1 & 43.00 & 42.88 & $-0.28 \%$ & & & & & 45.00 & 42.88 & \multicolumn{3}{|l|}{$-.28 \%$} & & \\
\hline 2 & 270.00 & 270.00 & $-0.00 \%$ & & & & & 283.00 & 282.33 & \multicolumn{3}{|l|}{$-0.24 \%$} & & \\
\hline 3 & 756.25 & 758.5 & $0.30 \%$ & & & & & 792.25 & 793.08 & \multicolumn{3}{|l|}{$0.10 \%$} & & \\
\hline 4 & 1481.25 & 1488.10 & $0.46 \%$ & & & & & 1553.00 & 1555.90 & \multicolumn{3}{|l|}{$0.19 \%$} & & \\
\hline \multicolumn{2}{|c|}{ Optimized E } & $1.7957 \mathrm{I}$ & $E+07$ & & & & & \multicolumn{2}{|c|}{ Optimized E } & \multicolumn{3}{|c|}{$1.96237 E+07$} & & \\
\hline \multicolumn{4}{|c|}{ Water RT } & \multicolumn{4}{|c|}{ Added Mass Frequency Factors } & \multicolumn{3}{|c|}{ LH2 } & \multicolumn{4}{|c|}{ Added Mass Frequency Factors } \\
\hline $\begin{array}{l}\text { Mode } \\
\text { (Test } \\
\text { Order) }\end{array}$ & Test $(\mathrm{Hz})$ & $\begin{array}{l}\text { ANSYS } \\
\text { Acoustic } \\
\text { FEM }\end{array}$ & $\%$ Error & Test & ANSY & $\left\{\begin{array}{l}\text { Lind- } \\
\text { holm }\end{array}\right.$ & Liang & Test (hz) & $\begin{array}{l}\text { ANSYS } \\
\text { Acoustic } \\
\text { FEM }(\mathrm{Hz})\end{array}$ & $\%$ Error & Test & ANSY & $\begin{array}{l}\text { Lind- } \\
\text { holm }\end{array}$ & Liang \\
\hline 1 & 25.25 & 25.46 & $0.82 \%$ & 0.587 & 0.594 & & & 42.50 & 42.12 & $-0.89 \%$ & 0.944 & 0.939 & & \\
\hline 2 & \begin{tabular}{|l|}
161.75 \\
\end{tabular} & 160.30 & $-0.90 \%$ & 0.599 & 0.594 & & & 267.50 & 265.37 & $-0.80 \%$ & 0.945 & 0.940 & & \\
\hline 3 & 459.75 & \begin{tabular}{|l|}
459.49 \\
\end{tabular} & $-0.06 \%$ & 0.608 & 0.606 & & & 750.75 & \begin{tabular}{|l|l|}
747.48 \\
\end{tabular} & $-0.44 \%$ & 0.948 & 0.943 & & \\
\hline \multirow[t]{2}{*}{4} & 911.00 & 918.34 & $0.81 \%$ & 0.615 & 0.617 & & & 1475.0 & 1471.50 & $-0.24 \%$ & 0.950 & 0.946 & & \\
\hline & & & & 0.602 & 0.603 & 0.622 & 0.597 & & & & 0.947 & 0.942 & 0.948 & 0.941 \\
\hline
\end{tabular}

\section{CONCLUSIONS}

The first natural frequency test of a cantilever beam in LH2 reported in the literature has been performed at NASA/MSFC to experimentally validate techniques for predicting the effect of the added mass of LH2. In addition, tests were performed at room temperature in air and in gas at close-to-cryogenic temperatures to enable an exhaustive correlation procedure that precisely generated values for Young's Modulus as a function of temperature. A final test of the beam in room temperature water was also performed. Based on the results, ANSYS $^{\circledR}$ acoustic modeling can be considered extremely accurate and can confidently be applied to more complex geometries. Our results for the Modulus of the Titanium alloy used in the test also fall well within the range predicted by proprietary sources. Both of these findings will prove extremely useful for the follow-on phase of this test-analysis program on the RS-25 inducer, and will be valuable for modeling of other structures in similar liquid cryogenic environments.

\section{REFERENCES}

1 Metallic Materials Properties Development and Standardization-08, Battelle Memorial Institute, 2013

2 Ghisi, A., Mariani,S., J., "Mechanical Characterization of Ti-5Al-2.5Sn ELI Alloy at Cryogenic and Room Temperatures", Int J. Fracture (2007) 146:61-77, doi 10.1007/s10704-007-9140-Z

3 Zhang, Z., Zhao, L.Z., Tu Z. H., Zhang, P.Q., "Dynamic Young's moduli of space materials at low temperatures," Cryogenics, 1994, Volume 34, Number 10, pp. 839-842

4 Lindholm, U, et. al., "Elastic Vibration Characteristics of Cantilever Plates in Water," Journal of Ship Research, June 1965, pp. 11-36

5 Ergin, A., Urgulu, B., "Linear vibration analysis of cantilever plate partially submerged in fluid", Journal of Fluids and Structures 17 (2003) 927-939,

6 Hosseini-Hashemi, S., Karimi, M., Rokni, H., "Natural Frequencies of rectangular Mindlin plates coupled with stationary fluid", Applied Mathematical Modelling 36 (2012) 764-778

7 Liang, Cho-C., Liao, Ching-C., Tai Yuh-S., Li, Wen-H., "The free vibration analysis of submerged cantilever plates", Ocean Engineering 28 (2001) 1225-1245

8 Kerboua, Y., et. al. "Vibration analysis of rectangular plates coupled with fluid", Applied Mathematical Modelling 32 (2008) 2570-2586 
9 Chiu, J., Brown, A. M.., "Characterization of the Modal Characteristics of Structures Operating in Dense Liquid Turbopumps,” ASME Gas Turbo Expo 2017, Charlotte, North Carolina, June 26-30, 2017, paper GT2017-63633 